\title{
Hardness of covering all the triangles
}

\author{
Thinh D. NguYeN* \\ \begin{tabular}{|l|} 
Moscow State University \\
kosmofarmer@gmail.com
\end{tabular} \\ July 17, 2018
}

\begin{abstract}
Vertex Cover and Edge Cover are two classical examples that are often used to show the contrast of problem solvability. While VerTEx Cover is hard, EDGE COVER can be solved in polynomial time. We claim that the former remains intractable even if the objects to be covered are triangles instead of edges. Therefore, one more combinatorial optimization problem, namely COVERING TRIANGLes, is added to the decades-old list of the problems in this research area.
\end{abstract}

\section{DEFINITIONS AND SKETCH OF PROOF}

If $G=(V, E)$ is a given graph, $H_{G}$ is a graph which has all of the vertices and edges of $G$, and also for each edge of $G$ like $e=(x, y), H_{G}$ has a new vertex which is connected to both $x$ and $y$.

A triangle is a complete graph with 3 vertices, also denoted $K_{3}$. Our goal is to prove that the problem of finding the minimum number of vertices to cover all the triangles in a given graph $G$ is $N P$-complete. It is worth mentioning that: (i) the Vertex Cover problem asks for a vertex cover that covers all edges, (ii) our problem Covering TRIANGLes problem also asks for a vertex cover but the covered objects are triangles, not edges. Hence, we call our problem Covering TRIANGLE to avoid misleading notation.

Sketch of Proof: We reduce from Vertex Cover of triangle-free cubic graph, Guruswami et al. prove the hardness of this problem in [2]. Our reduction proceeds in two steps,

\footnotetext{
*Perebor
} 
Step 1: Prove that for a given graph $G=(V, E), H_{G}$ has a vertex cover covering all the triangles with cardinality of $k$ vertices, iff. $G$ has a usual vertex cover set with $k$ vertices.

Step 2: Conclude that the problem of finding the smallest vertex cover to cover all the triangles in a given graph $G$ is $N P$-complete.

\section{Proving the Claim}

For an edge $e \in E(G)$, let $v^{e}$ denote the new vertex which we added to form $H_{G}$, that is, the new vertex which is connected to the endpoints of $e$. Assume that $v_{1}, \ldots, v_{k}$ is a usual vertex cover set of $G$. Then $\left\{v_{1}, \ldots, v_{k}\right\}$ is also covering all the triangles in $H_{G}$. To see this, remember that $G$ is triangle-free as we use the problem proved by Guruswami et al., so all the triangles in $H_{G}$ are the triangles with vertices $v_{e}$ connected with its corresponding edge $e$. Since our $k$-vertex cover of $G$ needs to cover each edge $e$ in $G$, it also leaves no triangles left in $H_{G}$.

It remains to show the reverse direction; namely, if $H_{G}$ has a $k$-vertex cover that covers all the triangles, then $G$ also has a usual $k$-vertex cover. Let $S \subseteq V\left(H_{G}\right)$ be its vertex cover covering all the triangles and $|S|=k$. We can decompose $S$ into $S_{1}=S \cap V(G)$ and $S_{2}=S \cap\left\{v^{e}: e \in E(G)\right\}$. Notice that if one of the two endpoints of $e$ belongs to $S_{1}$ ), then $v^{e} \notin S_{2}$, otherwise $v^{e}$ is redundant in $S$. As a result, for each $v^{e} \in S_{2}$, we can replace it in $S$ by any unused vertex of $e$. Having done these replacements, the cardinality of $S$ stays the same, while no $v_{e}$ remains in $S$ anymore. We claim that $S$ is now also a usual vertex cover of $G$. Indeed, let $e$ be any edge of $G$. Assume to the contrary that $S$ does not cover $e$, the two endpoints of $e$ do not belong to $S$. To cover the triangle corresponding to this edge, $S$ must include $v_{e}$. But, we have done the aforementioned replacement and claimed that $S$ has no $v^{e}$ left. So, $S$ must already cover $e$. We conclude that $S$ is a usual vertex cover of G.Q.E.D

\section{CONCLUSION}

As long as we do the research on a well-known conjecture, we should recall our mathematical nature from Kvant, Prasolov-style of doing mathematics, similar to mathematics of [3] back to those beautiful days.

\section{REFERENCES}

[1] Michael R. Garey, David S. Johnson, Computers and Intractability: A Guide to the Theory of NP-Completeness 
[2] Venkatesan Guruswami, C. Pandu Rangan, M. S. Chang, G. J. Chang, and C. K. Wong, The Vertex-Disjoint Triangles Problem, Springer-Verlag in Berlin, Heidelberg, 1998

[3] Phan Dinh Dieu, Le Cong Thanh, Le Tuan Hoa, Average Polynomial Time Complexity of Some NP-Complete Problems. Theor. Comput. Sci. 46(3): 219-237 (1986) 